BNL--47969

DE93 001656

\title{
End Effects and Orbit Correction in the Wiggler for the ATF Harmonic Generation FEL *
}

\author{
X. Zhang, I. Ben-Zvi, G. Ingold, S. Krinsky, and L. H. Yu \\ National Synchrotron Light Source \\ Brookhaven National Laboratory \\ Upton, NY 11973
}

\begin{abstract}
In this paper, we consider the superconducting wiggler magnet under construction for the High Gain Harmonic Generation experiment (HGHG) at the Accelerator Test Facility (ATF) at BNL. We analyze the effects due to the ends of the wiggler and discuss their correction. In addition, the localized field produced by a trim coil for horizontal beam steering is investigated.
\end{abstract}

\section{Introduction}

A superconducting electromagnetic wiggler shown in Fig. 1 has been developed [1] for the HGHG experiment at the ATF at BNL [2]. The $156.6 \mathrm{~cm}$ long wiggler has a total of 86 periods. The period length is $18 \mathrm{~mm}$ and the full gap of the wiggler is $8 \mathrm{~mm}$. A parabolic shape cut at the inside surface of the wiggler pole provides equal focussing in both horizontal and vertical planes. The ferromagnetic yoke of the wiggler is machined out of a solid block of low carbon steel. A superconducting $\mathrm{NbTi}$ coil is wound continuously along the yoke, with the winding direction alternating every half period. The wiggler is under construction by a collaboration between BNL and Grumman. The advantages of this superconducting wiggler are:

a) high magnetic field;

b) easy tuning;

c) two-dimension focussing;

d) high precision of the magnetic field distribution.

- This work was performed under the auspices of the U.S. Department of Energy. 


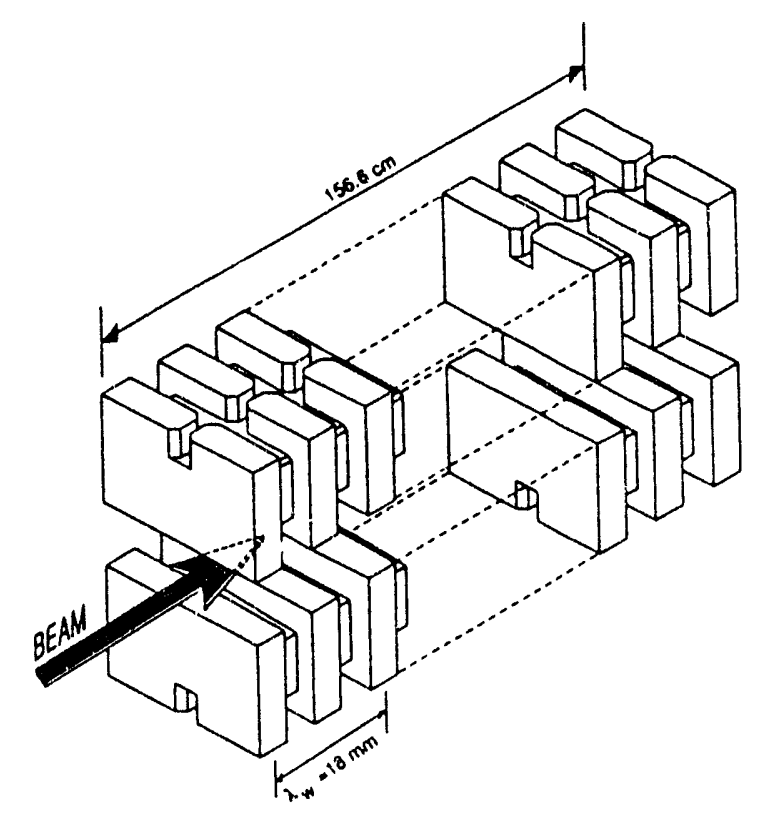

Figure 1: The superconducting wiggler presently under construction.

In this paper, we present an analysis of the wiggler end effects and the design of a trim coil in the wiggler to provide a localized field to correct the electron trajectory. In section II, we begin with the discussion of the ideal periodic wiggler with infinite periods. Based on this ideal case, we examine the effects due to the end of the wiggler and discuss their compensation in section III. We then turn to the study of generating a localized field distribution for particle orbit correction in section IV.

\section{Performance of the Ideal Periodic Wiggler}

It is worthwhile to have a brief discussion of the performance of the wiggler introduced in section I with an infinite number of periods before dealing with the problems of end effects and the trim coil. In the case of periodic boundary condition and finite permeability, a sine-like field is generated with the period equal to the wiggler wavelength. In the mid-plane, the peak field is $0.578 \mathrm{~T}$ for a current of $6 \mathrm{kA}$-turns. The field line plot generated by POISSON is shown in Fig. 2, and the field in the mid-plane is shown in Fig. 3.

The peak field vs the excited current is plotted in Fig. 4. Clearly, the wiggler starts saturating when the current becomes higher than $3 \mathrm{kA}$-turns. The HGHG experiment is designed to work with a peak field of $5.8 \mathrm{k}$-Gauss, which corresponds to a current of about $6 \mathrm{kA}$-turns. In this case the saturation reduces the field by about $24 \%$. 


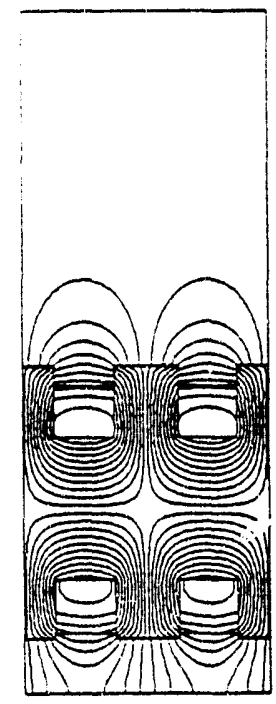

Figure 2: POISSON field line plots of the wiggler with periodic boundary conditions.

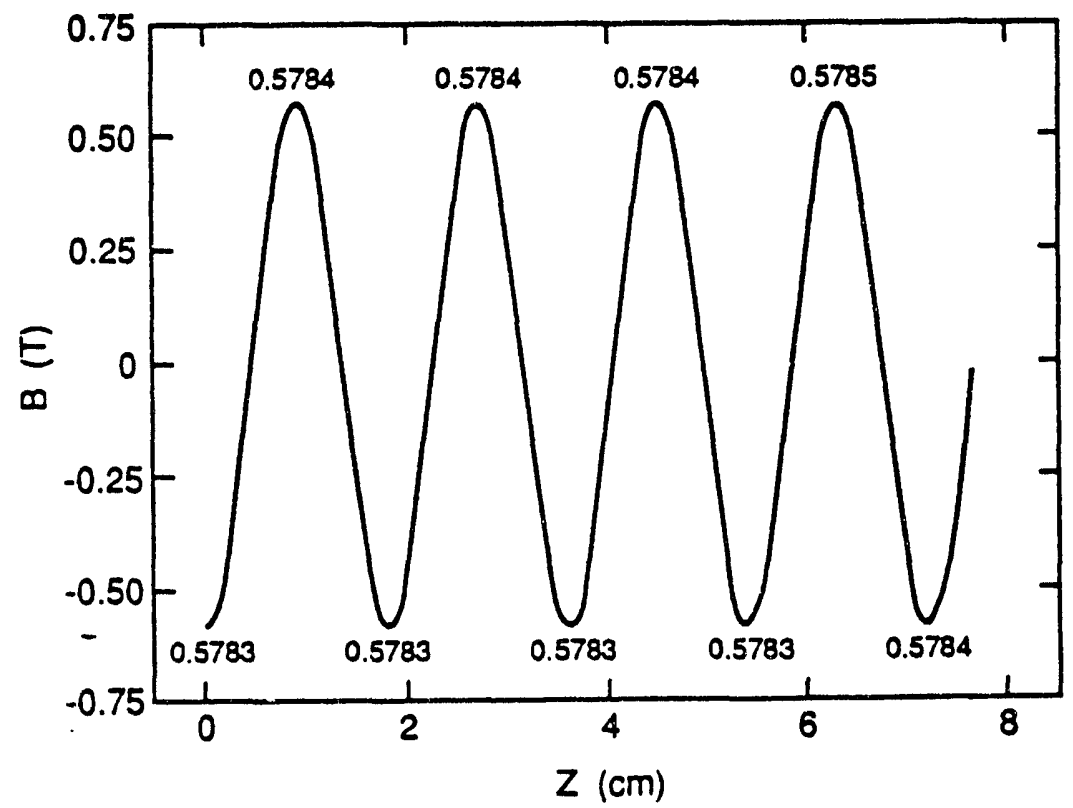

Figure 3: Mid-plane field vs longitudinal distance. 


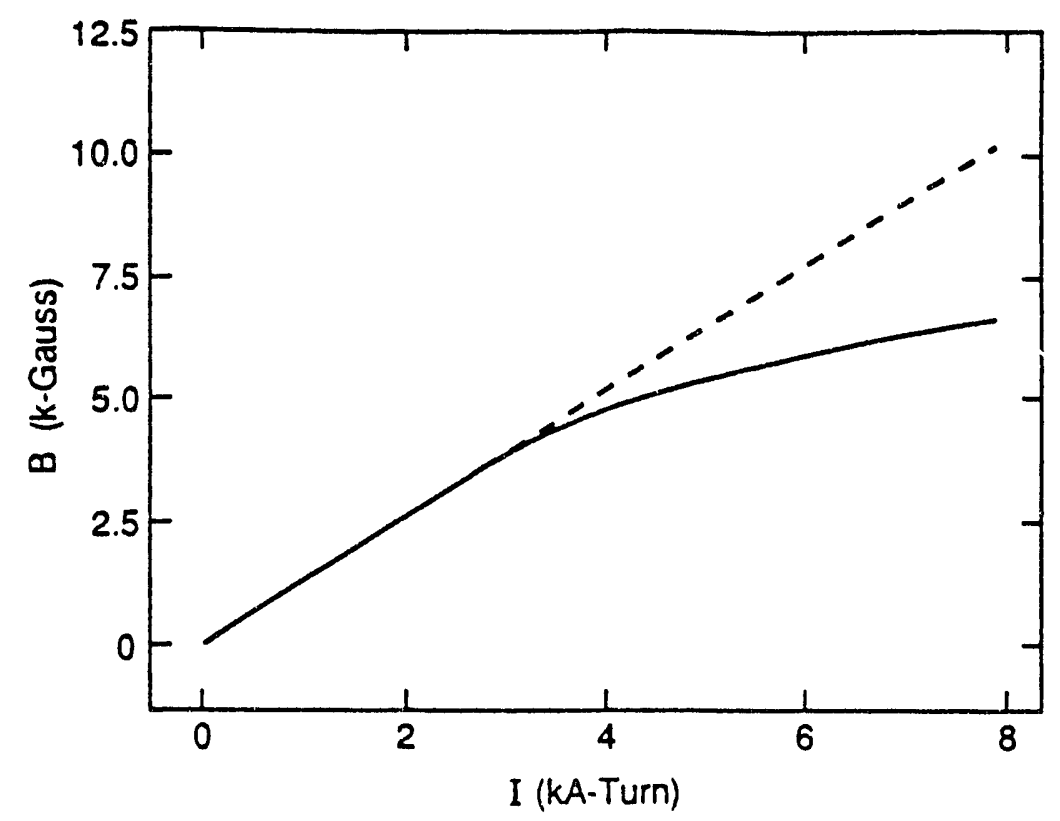

Figure 4: The peak magnetic field vs excited current.

\section{Wiggler End Effects}

To reduce the angular and displacement errors, we use a binomial excitation pattern [3] of order 3 , i.e., the coils are excited with a pattern of $1:-3: 4:-4: 4:-4: \cdots$, starting from the end of the wiggler. This compensation scheme requires the wiggler to have an odd number of poles and an even number of coils. The fictitious iron bar $[4,5]$ with a large constant permeability $\left(\mu=1 \times 10^{5}\right)$ connecting the end pole to the left boundary is simulating a magnetic field clamp. The field line plot generated by POISSON without correction is shown in Fig. 5. Note that there are a few field line passing through the clamp. The field in the mid-plane is shown in Fig. 6. Due to the end effect, the field is seen to reach its equilibrium value after 1 to 2 wiggler periods. Due to saturation, the first field integral is about 650 Gauss-cm for a current of 6 kA-turns (equivalent to giving $6.5 \mathrm{mrad}$ angular kick) in spite of binomial excitation pattern.

To compensate the angular kick, a pair of extra correction coils are added on each side of the first field pole. When the current in these coils are adjusted to 240 A-turns (the current initially is $1500 \mathrm{~A}$-turns in the first coil), the first field integral essentially vanishes. The field lines and the field in the mid-plane including the correction are plotted in Figs. 7 and 8, respectively. With the currection, there are no field lines passing through the field clamp. As we expected, the correction coils mainly affect the fields in the region of first and second poles making the the first field integral vanish. In addition, a nonzero second field integral is seen, which is displayed in Fig. 9. Clearly, the electron trajectory will have a constant displacement 


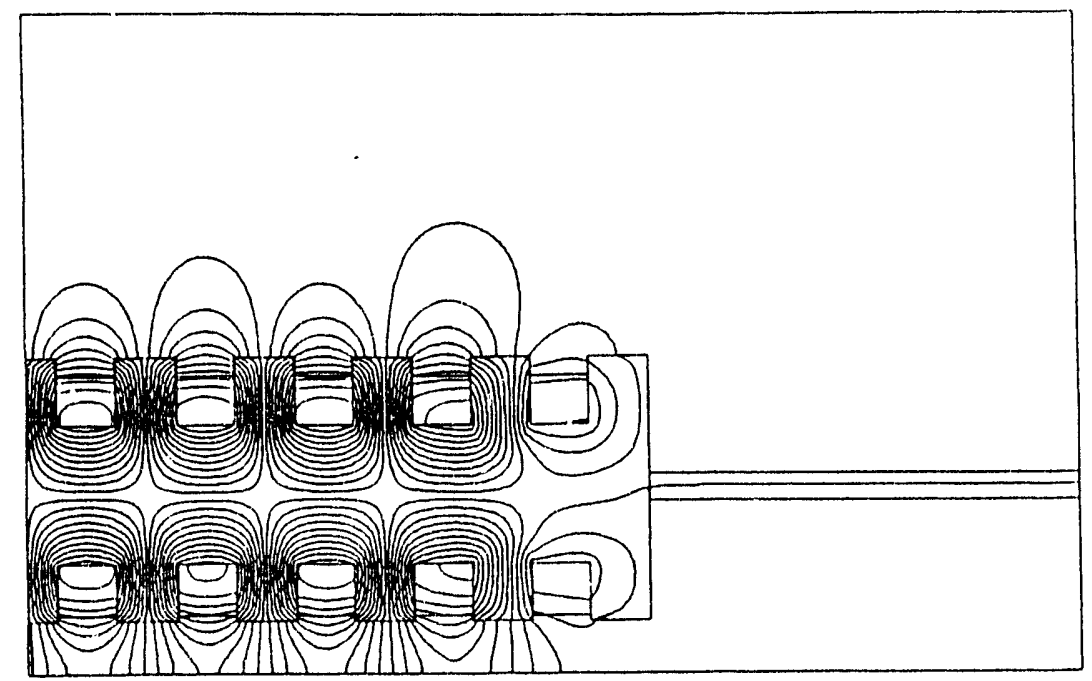

Figure 5: POISSON field line plots without compensation.

associated with this second integral when it goes through the wiggler end. In the case with compensation, the second integral is about 1000 Gauss $-\mathrm{cm}^{2}$ and it can introduce about $0.1 \mathrm{~mm}$ centroid shift for our $30 \mathrm{MeV}$ beam.

To reduce the displacement associated with the second integral, the 4 th binomial excitation may be used, with a pattern of $1:-4: 7:-8: 8:-8: \cdots$ starting from the end of the wiggler. To have this pattern, the wiggler needs to contain an even number of poles and an odd number of coils. The scalar pole potentials with this pattern are $1:-3: 4:-4: 4: \ldots$. The field along the $z$-axis is anti-symmetric and the potential is symmetric about the center coil, which is different from the case of order 3 , where the field is symmetric and the potential is antisymmetric about the center pole. Using a POISSON simulation in the regime of low saturation, we have verified that this pattern is steering-free and displacement-free as expected.

\section{Design of Trim Coil}

Errors in the wiggler magnetic field may cause an electron beam centroid shift (transversely) and an electron phase deviation (longitudinally). In either case, the errors may reduce FEL gain and radiated power. Since errors in the real wiggler are inevitable, their correction is necessary. Furthermore, in the case of the HGHG experiment, the latter part of the second wiggler is tapered for maximum energy extraction from electron beam. The steering error in the transition between wiggler sections with unequal currents is minimized using the 3 rd order binomial coefficient in each transition. This angular error can reach $0.3 \mathrm{mrad}$ for $30 \mathrm{Mev}$ beam. The correctors 


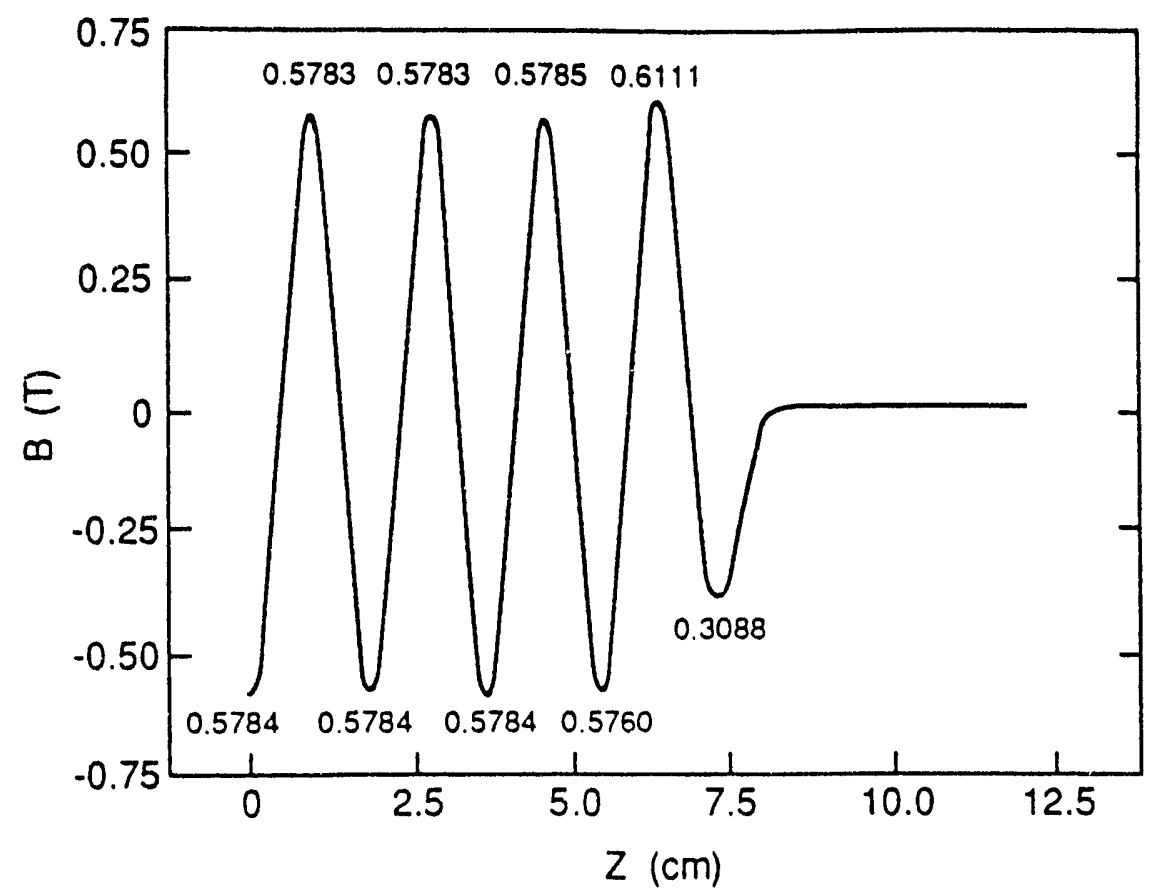

Figure 6: Mid-plane field vs longitudinal distance without compensation.

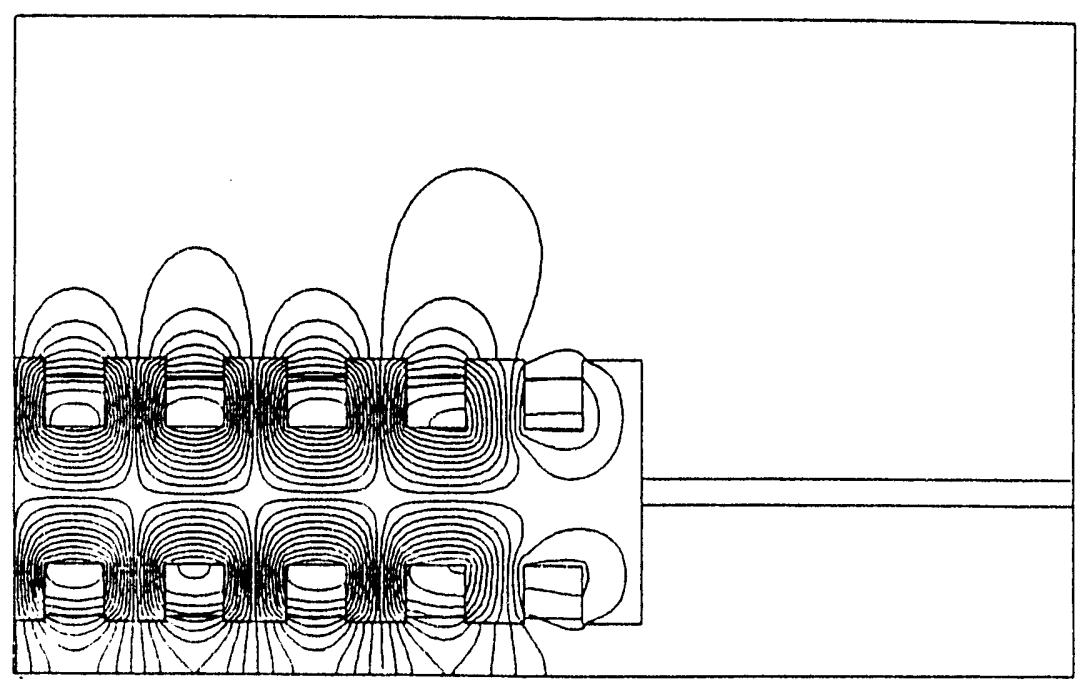

Figure 7: POISSON field line plots with compensation. 


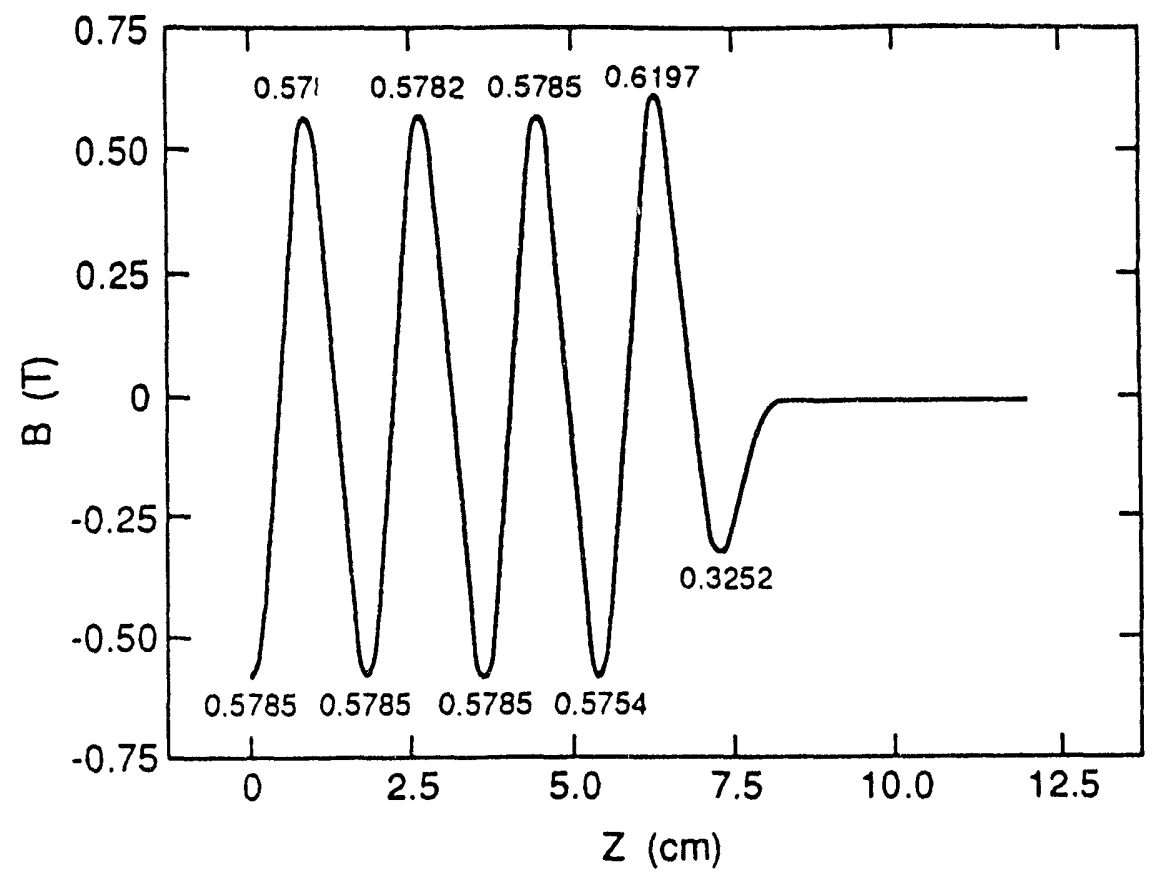

Figure 8: Mid-plane field vs longitudinal distance with compensation.

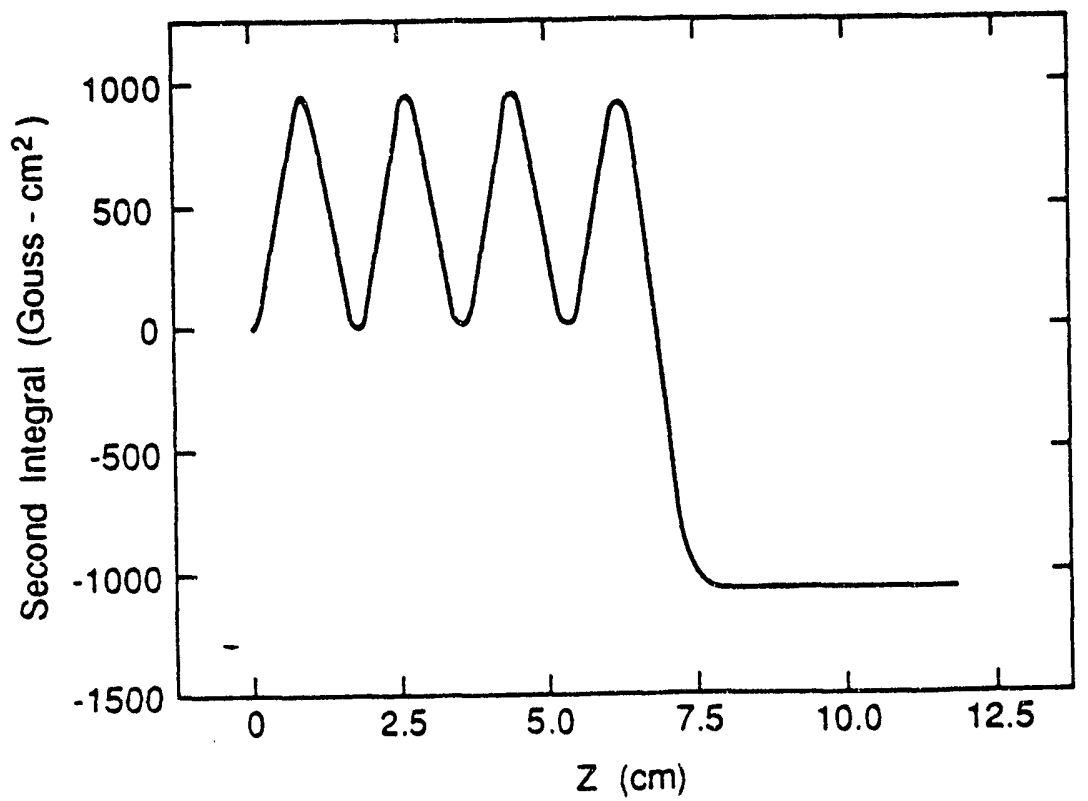

Figure 9: Field second integral in the mid-plane vs longitudinal distance with compensation. 
along the wiggler are conservatively designed to provide about $3 \mathrm{mrad}$ angular kick.

A magnetic correction produced by a trim coil is suitable for the correction in the superconducting wiggler. The trim coil is superimposed on the main coil in the wiggler. For $3 \mathrm{mrad}$ angular kick, the trim coil should generate a localized magnetic field with a field integral of 300 Gauss-cm. To eliminate the change in the trajectory length due to the trim coil, the trim coil should cover an even number of poles [4]. The change in trajectory length $\Delta s(z)$ can be divided into three parts [4],

$$
\Delta S(z)=x_{0}(z) \Delta x^{\prime}(z)-\int x_{0}(z) \Delta x^{\prime \prime}(z) d z+\frac{1}{2} \int \Delta x^{\prime 2}(z) d z
$$

Here, $x_{0}$ is particle horizontal position given by the original wiggler magnetic field, $\Delta x^{\prime}$ and $\Delta x^{\prime \prime}$ are the angular kick and its derivative respectively, which are introduced by the field increment $\Delta B$ produced by trim coil. The first term vanishes if evaluated at the positions of integer number of the periods. If the field increment $\Delta B$ is symmetric with $z$-coordinate and $x_{0}$ is anti-symmetric, and if the integral is carried out over an integer number of wiggler periods, then the second term vanishes. The last term always exists since the integrand is definite positive. However, this term may provide a canceliation of similar term introduced by the wiggler errors, which the trim coil is designed to correct.

The irim coil is powered by 100 Ampere-turns and covers 2 poles. The field produced by the trim coil has very local effects, which mainly act on poles covered by the trim coil as shown in Fig. 10. With 100 A-turns the first field integral in the trim coils is close to 300 Gauss-cm. In the region of small number of ampere-turns, we find that the field integral almost linearly increases with either the number of ampere-turns or the number of poles to be covered by the trim coil. To ensure the proper positioning of the electron beam centroid in the HGHG experiment, 6 trim coils will be set along the superconducting wiggler with equal spacing. Each of them will cover 4 poles to reduce the driving power for the trim coil currert.

\section{ACKNOWLEDGMENTS}

We express our thanks to $\mathrm{K}$. Halbach for his suggestions, discussions and comments. 


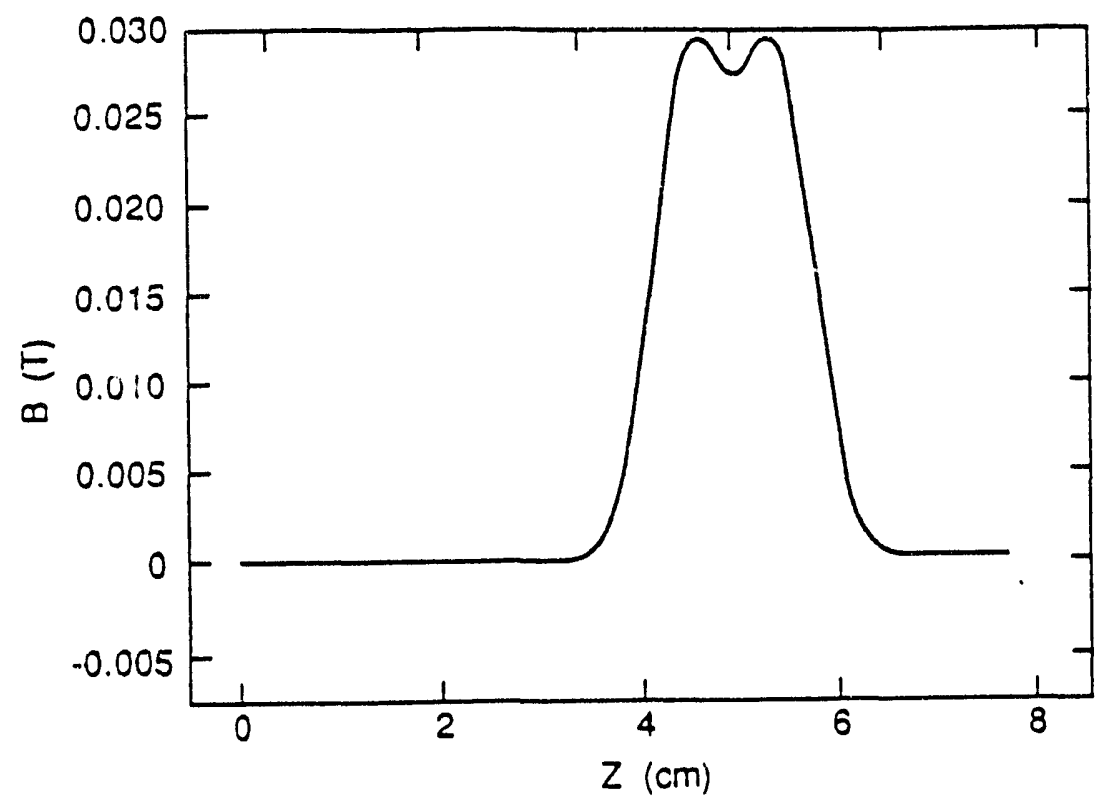

Figure 10: Mid-plane field generated by trim coil vs longitudinal distance.

\section{References}

[1] I. Ben-Zvi, et al., Proc. of 1991 Inte. FEL Conf., Santa Fe, NM.

[2] I. Ben-Zvi, et al., Proc. of 1991 Inte. FEL Conf., Santa Fe, NM.

[3] K. Ha'bach, Nucl. Inst. \& Meth. A250 95(1986).

[4] K. Halbach, Private communication.

[5] X. Zhang, et al., to appear in BNL Report.

\section{DISCLAIMER}

This report was prepared as an account of work sponsored by an agency of the United States Government. Neither the United States Gnvernment nor any agency thereof, nor any of their employees, makes any warranty, express or implied, or assumes any legal liability or responsibility for the accuracy, completeness, or usefulness of any information, apparatus, product, or process disclosed, or represents that its use would not infringe privately owned rights. Reference herein to any specific commercial product, process, or service by trade name, trademark, manufacturer, or otherwise does not necessarily constitute or imply its endorsement, recommendation, or favoring by the United States Government or any agency thereof. The views and opinions of authors expressed herein do not necessarily state or reflect those of the United States Government or any agency thereof. 

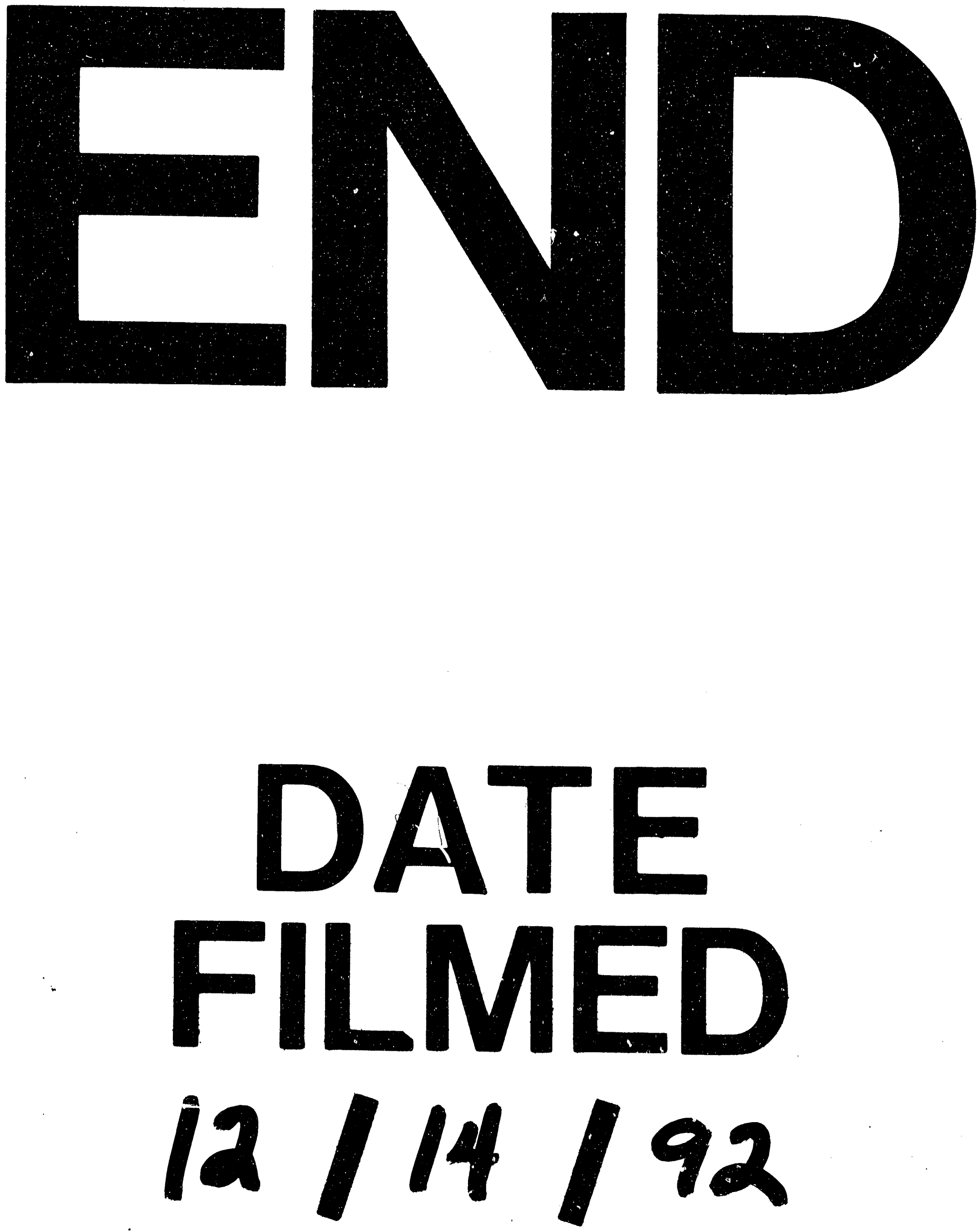
